\title{
Parameterization of the Box Variety by Theta Functions
}

\author{
Eberhard Freitag \& Riccardo Salvati Manni
}

\section{Introduction}

We consider the graded algebra (the generators have weight one)

$$
B=\mathbb{Q}\left[Z_{1}, Z_{2}, Z_{3}, W_{1}, W_{2}, W_{3}, C\right]
$$

with defining relations

$$
\begin{aligned}
W_{1}^{2}+W_{2}^{2} & =Z_{3}^{2}, \\
W_{1}^{2}+W_{3}^{2} & =Z_{2}^{2}, \\
W_{2}^{2}+W_{3}^{2} & =Z_{1}^{2}, \\
W_{1}^{2}+W_{2}^{2}+W_{3}^{2} & =C^{2} .
\end{aligned}
$$

This is a normal graded algebra. The associated projective variety $\operatorname{proj}(B)$ is called the box variety or variety of cuboids. It is absolutely irreducible. We denote its complexification by

$$
\mathcal{B}:=\operatorname{proj}\left(B \otimes_{\mathbb{Q}} \mathbb{C}\right) .
$$

It is a surface that characterizes cuboids. The variables $W_{i}$ give the edges of the cuboid, the variables $Z_{i}$ the diagonals of the faces, and $C$ the long diagonal. There is an unsolved problem, raised by Euler, whether the box variety contains nontrivial rational points or not. For more details on the box variety, we refer to $[v L]$ and [ST].

In this note, we describe a parameterization of the box variety by theta functions. This will imply that it is a quotient of the product $\overline{\mathbb{H} / \Gamma[8]} \times \overline{\mathbb{H} / \Gamma[8]}$ of two modular curves of level 8 by a group of order 8 that comes from the diagonal action of $\Gamma[4]$. In fact, this parameterization can be defined over the cyclotomic field $K=\mathbb{Q}\left(\zeta_{8}\right)=\mathbb{Q}(\mathrm{i}, \sqrt{2})$. We found this parameterization from an observation of D. Testa that the box variety can be embedded into a certain Siegel modular variety, which has been described by van Geemen and Nygaard. This background is not necessary for our note, and we will not describe it here. But we want to point out that the still unpublished work [ST] is behind the scenes, and we are very grateful that he explained to us details of this work.

This parameterization can be used to derive quickly known properties and also some new ones of the box variety. For example, we give in Section 2 a modular description of the automorphism group. It can be realized as a subgroup of $\operatorname{SL}(2, \mathbb{Z}) \times \operatorname{SL}(2, \mathbb{Z})$. In $[S T]$ and $[v L], 140$ rational and elliptic curves on the minimal model of the box variety that give the generators of the Picard group have

Received July 26, 2013. Revision received September 7, 2016. 
been described. We describe them in Section 3 in a very simple way as certain modular curves.

In Section 3, we consider smooth curves in the box variety. We prove an estimate that shows how their genus grows with their degree. As a consequence, smooth rational and elliptic curves have a bounded degree. This can be considered as a supporting evidence of the conjecture made in [ST] that the 140 curves described in [vL] exhaust all rational and elliptic curves. This has been proved in [ST] for degrees $\leq 4$.

In the paper [Be], the box variety arises as a member of a whole family having the same properties, namely to be complete intersections of 4 quadrics in $\mathbb{P}^{6}$ with an even set of 48 nodes. In this paper, Beauville also describes a certain smooth two-fold Galois covering $X$ of the box variety. It is unramified outside the 48 nodes, and it is a minimal surface of general type with $q=4, p_{g}=7$, and $K^{2}=32$. In Section 4, we give a very simple modular description of it.

In Section 5, we consider a certain involution $\sigma$ of the box variety $\mathcal{B}$. We use the modular description to realize the quotient $\mathcal{B} / \sigma$ as a Kummer variety.

In the last section, we consider a certain moduli problem that gives the realization of the box variety as a fine moduli scheme over $\mathbb{Q}(i)$ classifying pairs $(E, F)$ of elliptic curves with level 4 structures and a compatible isomorphism $E[8] \rightarrow F[8]$. This is closely related to work of Kani and Schanz [KS].

We want to thank A. Beauville, E. Kani, and D. Testa for helpful discussions. We thank the referee for pointing out a serious gap in the first version of the paper and for his hint how to overcome this problem.

\section{Generalities about Modular Groups}

We use the standard notations

$$
\Gamma[N]=\operatorname{kernel}(\operatorname{SL}(2, \mathbb{Z}) \longrightarrow \operatorname{SL}(2, \mathbb{Z} / N \mathbb{Z}))
$$

for the principal congruence subgroup of level $N$ of the elliptic modular group and

$$
\begin{aligned}
& \Gamma_{0}[N]=\left\{M=\left(\begin{array}{ll}
a & b \\
c & d
\end{array}\right) \in \operatorname{SL}(2, \mathbb{Z}) ; c \equiv 0 \bmod N\right\}, \\
& \Gamma_{1}[N]=\left\{M=\left(\begin{array}{ll}
a & b \\
c & d
\end{array}\right) \in \operatorname{SL}(2, \mathbb{Z}) ; a \equiv b \equiv 1 \bmod N, c \equiv 0 \bmod N\right\} .
\end{aligned}
$$

We also will use the Igusa groups

$$
\Gamma[N, 2 N]=\left\{M=\left(\begin{array}{ll}
a & b \\
c & d
\end{array}\right) \in \Gamma[N] ; a b \equiv c d \equiv 0 \bmod 2 N\right\} .
$$

In the following, we define the square root $\sqrt{a}$ of a nonzero complex number by the principal part of the logarithm. This means that the real part is positive if $a$ is not real and negative and that the imaginary part is positive if $a$ is real and negative. Let $\Gamma \subset \operatorname{SL}(2, \mathbb{Z})$ be a subgroup of finite index, and let $r$ be an integer. 
By a multiplier system of weight $r / 2$ we understand a map $v: \Gamma \rightarrow S^{1}$ such that

$$
v(M) \sqrt{c \tau+d}^{r}, \quad M=\left(\begin{array}{ll}
a & b \\
c & d
\end{array}\right),
$$

is a one-cocycle. Then the space $[\Gamma, r / 2, v]$ of holomorphic modular forms can be defined in the usual way. Their transformation law is

$$
f(M \tau)=v(M) \sqrt{c \tau+d}^{r} f(\tau) .
$$

There are two basic multiplier systems. The theta multiplier system $v_{\vartheta}$ is a multiplier system of weight $1 / 2$ on the theta group

$$
\Gamma_{\vartheta}:=\Gamma[1,2] \text {. }
$$

It can be defined as the multiplier system of the theta function

$$
\vartheta(\tau)=\sum_{m=-\infty}^{\infty} e^{\pi \mathrm{i} n^{2} \tau} .
$$

The theta group is generated by $\left(\begin{array}{ll}1 & 2 \\ 0 & 1\end{array}\right)$ and $\left(\begin{array}{cc}0 & -1 \\ 1 & 0\end{array}\right)$. From the theta inversion formula $\vartheta(-1 / \tau)=\sqrt{\tau / \mathrm{i} \vartheta}(\tau)$ we get

$$
v_{\vartheta}\left(\begin{array}{ll}
1 & 2 \\
0 & 1
\end{array}\right)=1, \quad v_{\vartheta}\left(\begin{array}{cc}
0 & -1 \\
1 & 0
\end{array}\right)=e^{-\pi \mathrm{i} / 4} .
$$

We also have to consider the theta function of the second kind $\Theta(\tau):=\vartheta(2 \tau)$. This is a modular form for $\Gamma_{0}[4]$. We denote its multiplier system by $v_{\Theta}$. Both multiplier systems $v_{\vartheta}$ and $v_{\Theta}$ agree on $\Gamma[8]$. This follows from classical theta transformation formulae. Details have been worked out in [Kl] or [vGN].

For given $\Gamma, r_{0}$, and $v$, we can consider the graded algebra

$$
A\left(\Gamma, r_{0}, v\right):=\sum_{r \in \mathbb{Z}}\left[\Gamma, r r_{0}, v^{r}\right] .
$$

If it is clear which $\left(r_{0}, v\right)$ has to be considered, we will simply write $A(\Gamma)$ for this algebra. This is a finitely generated algebra of Krull dimension 2. We know (see [Fr2]) that the associated projective curve $\operatorname{proj}(A(\Gamma))$ is the Satake compactification

$$
\overline{\mathbb{H} / \Gamma}=\mathbb{H}^{*} / \Gamma \quad \text { where } \mathbb{H}^{*}=\mathbb{H} \cup \mathbb{Q} \cup\{\infty\} .
$$

We have to consider more generally subgroups of finite index $\Gamma \subset \operatorname{SL}(2, \mathbb{Z}) \times$ $\operatorname{SL}(2, \mathbb{Z})$. A multiplier system $v$ of weight $r / 2$ now means a function $v: \Gamma \rightarrow S^{1}$ such that

$$
v\left(M_{1}, M_{2}\right){\sqrt{c_{1} \tau+d_{1}}}^{r}{\sqrt{c_{2} \tau+d_{2}}}^{r}
$$

is a one-cocycle. The spaces of modular forms $[\Gamma, r / 2, v]$ (now functions of two variables) and the algebras $A(\Gamma)=A\left(\Gamma, r_{0}, v\right)$ are defined in the obvious way.

Let $N$ be a divisor of the natural number $N^{\prime}$. In this paper, the group

$$
\Delta\left(N, N^{\prime}\right)=\left\{\left(M_{1}, M_{2}\right) \in \Gamma[N] \times \Gamma[N], M_{1} \equiv M_{2} \bmod N^{\prime}\right\}
$$

plays an important role. It is generated by $\Gamma\left[N^{\prime}\right] \times \Gamma\left[N^{\prime}\right]$ and the diagonally embedded $\Gamma[N]$. 


\section{A Parameterization of the Box Variety by Theta Functions}

We make use of the Jacobi theta functions

$$
\vartheta_{a, b}(z)=\sum_{n=-\infty}^{\infty} e^{\pi \mathrm{i}\left((n+a / 2)^{2} z+b(n+a / 2)\right)} .
$$

Here $(a, b)$ is one of the three pairs $(0,0),(1,0),(0,1)$. These three functions are modular forms of weight $1 / 2$ with respect to the three conjugate groups of the theta group contained in $\operatorname{SL}(2, \mathbb{Z})$. The multiplier systems agree on the group $\Gamma[4,8]$ with $v_{\vartheta}$. We also consider the two theta functions of the second kind $\vartheta_{00}(2 \tau)$ and $\vartheta_{10}(2 \tau)$. They are modular forms for the two conjugated groups of $\Gamma_{0}[4]$. Their multiplier systems agree on $\Gamma[2,4]$ with $v_{\Theta}$.

So we see that the five functions

$$
\vartheta_{00}(z), \quad \vartheta_{10}(z), \quad \vartheta_{01}(z), \quad \vartheta_{00}(2 z), \quad \vartheta_{10}(2 z)
$$

have the same multiplier system on $\Gamma[8]$. Hence, they are contained in the ring

$$
A(\Gamma[8]):=\bigoplus_{r \in \mathbb{Z}}\left[\Gamma[8], r / 2, v_{\vartheta}^{r}\right] .
$$

It is not difficult to show the following result. The details have been worked out in the Heidelberg Diplomarbeit $[\mathrm{Br}]$.

THEOREM 2.1. We have

$$
A(\Gamma[8])=\mathbb{C}\left[\vartheta_{00}(z), \vartheta_{10}(z), \vartheta_{01}(z), \vartheta_{00}(2 z), \vartheta_{10}(2 z)\right] .
$$

Defining relations are the classical theta relations

$$
\begin{aligned}
& \vartheta_{00}(z)^{2}=\vartheta_{00}(2 z)^{2}+\vartheta_{10}(2 z)^{2}, \\
& \vartheta_{01}(z)^{2}=\vartheta_{00}(2 z)^{2}-\vartheta_{10}(2 z)^{2}, \\
& \vartheta_{10}(z)^{2}=2 \vartheta_{00}(2 z) \vartheta_{10}(2 z) .
\end{aligned}
$$

A quick way to see this is to use the fact that the defined variety is a smooth complete intersection. From this it follows easily that the ring defined by these relations is a normal ring of Krull dimension 2. Hence, the relations are defining relations. We can compute the Hilbert polynomial and compare it with well-known dimension formulae for spaces of modular forms.

Since the multiplier system $v_{\vartheta}$ is defined on the theta group $\Gamma_{\vartheta}$, we can define an action of the theta group on $A(\Gamma[8])$ by the formula

$$
f \mid M(\tau)=v_{\vartheta}(M)^{-r} \sqrt{c z+d}^{-r} f(M z) .
$$

This is an action from the right, $f\left|\left(M_{1} M_{2}\right)=\left(f \mid M_{1}\right)\right| M_{2}$. We describe it by means of matrices. For this, we have to use the action of $\operatorname{GL}(n, \mathbb{C})$ on a complex vector space $V$ with basis $e_{1}, \ldots, e_{n}$ from the right. It is defined by $A e_{i}=$ $\sum a_{i j} e_{j}$. If we write an element of $V$ in the form $\sum x_{i} e_{i}$, then this means that the row $x=\left(x_{1}, \ldots, x_{n}\right)$ has to be multiplied from the right by the matrix $A$. Standard theta transformation formulas give the following result. 
LEMMA 2.2. The matrix $\left(\begin{array}{ll}1 & 2 \\ 0 & 1\end{array}\right)$ acts with respect to the basis

$$
\vartheta_{00}(z), \quad \vartheta_{10}(z), \quad \vartheta_{01}(z), \quad \vartheta_{00}(2 z), \quad \vartheta_{10}(2 z)
$$

through the diagonal matrix with the diagonal entries

$$
1, \mathrm{i}, 1,1,-1 \text {. }
$$

The matrix $\left(\begin{array}{cc}0 & -1 \\ 1 & 0\end{array}\right)$ acts with respect to this basis through the matrix

$$
\left(\begin{array}{ccccc}
1 & 0 & 0 & 0 & 0 \\
0 & 0 & 1 & 0 & 0 \\
0 & 1 & 0 & 0 & 0 \\
0 & 0 & 0 & 1 / \sqrt{2} & 1 / \sqrt{2} \\
0 & 0 & 0 & 1 / \sqrt{2} & -1 / \sqrt{2}
\end{array}\right) .
$$

We are interested in the action of $\Gamma[4]$ on $A(\Gamma[8])$. The factor group $\Gamma[4] / \Gamma[8]$ is isomorphic to $(\mathbb{Z} / 2 \mathbb{Z})^{3}$. It is generated by the images of the matrices

$$
T=\left(\begin{array}{ll}
1 & 4 \\
0 & 1
\end{array}\right), \quad T^{\prime}=\left(\begin{array}{ll}
1 & 0 \\
4 & 1
\end{array}\right), \quad R=\left(\begin{array}{cc}
5 & 8 \\
8 & 13
\end{array}\right) .
$$

From Lemma 2.2 we get the following result.

LEMMA 2.3. The generators $T, T^{\prime}, R$ of $\Gamma[4] / \Gamma[8]$ act on $A(\Gamma[8])$ by means of the diagonal matrices

$$
\begin{aligned}
T & \longmapsto \operatorname{diag}(1,-1,1,1,1), \\
T^{\prime} & \longmapsto \operatorname{diag}(1,1,-1,1,1), \\
R & \longmapsto \operatorname{diag}(1,1,1,-1,-1) .
\end{aligned}
$$

Now we consider modular forms of two variables. We consider the ring $A(\Gamma[8] \times$ $\Gamma[8])$ of modular forms of integral or half integral weight $r / 2$ with respect to the multiplier system $\left(v_{\vartheta}\left(M_{1}\right) v_{\vartheta}\left(M_{2}\right)\right)^{r}$. It is easy to show, using Theorem 2.1 and a standard argument of Krull (compare the proof of III, $1.4_{1}$ in [Fr2]), that

$$
\begin{aligned}
& A(\Gamma[8] \times \Gamma[8])=\mathbb{C}[f(z) g(w)], \\
& \quad f, g \in\left\{\vartheta_{00}(\cdot), \vartheta_{10}(\cdot), \vartheta_{01}(\cdot), \vartheta_{00}(2 \cdot), \vartheta_{10}(2 \cdot)\right\} .
\end{aligned}
$$

We want to determine the subring $A(\Delta(4,8))$ of modular forms with respect to the group $\Delta(4,8)$. This is the ring of invariants with respect to the diagonal action of $\Gamma[4]$ by means of the action

$$
f(z, w) \longmapsto v_{\vartheta}(M)^{-2 r} \sqrt{c z+d}^{-r} \sqrt{c w+d}^{-r} f(M z, M w) .
$$

By Lemma 2.3 it is obvious that the forms

$$
\begin{aligned}
& \vartheta_{00}(z) \vartheta_{00}(w), \\
& \vartheta_{10}(z) \vartheta_{10}(w), \\
& \vartheta_{01}(z) \vartheta_{01}(w), \\
& \vartheta_{00}(2 z) \vartheta_{00}(2 w), \\
& \vartheta_{00}(2 z) \vartheta_{10}(2 w),
\end{aligned}
$$




$$
\begin{aligned}
& \vartheta_{10}(2 z) \vartheta_{00}(2 w), \\
& \vartheta_{10}(2 z) \vartheta_{10}(2 w)
\end{aligned}
$$

are invariant under the action of the quotient $\Gamma[4] / \Gamma[8]$. Moreover, since the action of the group is twisting by a character, we can show that they generate the invariant ring. In fact, the action of the group $A(\Gamma[8] \times \Gamma[8])$ depends on the multipliers and eventually on a sign.

THEOREM 2.4. There is an isomorphism

$$
B \otimes_{\mathbb{Q}} \mathbb{C} \stackrel{\sim}{\longrightarrow} A(\Delta(4,8)),
$$

which is given by

$$
\begin{aligned}
Z_{1} & \longmapsto \vartheta_{01}(z) \vartheta_{01}(w), \\
Z_{2} & \longmapsto \vartheta_{00}(z) \vartheta_{00}(w), \\
Z_{3} & \longmapsto \vartheta_{10}(z) \vartheta_{10}(w), \\
C & \longmapsto \vartheta_{00}(2 z) \vartheta_{00}(2 w)+\vartheta_{10}(2 z) \vartheta_{10}(2 w), \\
W_{1} & \longmapsto \vartheta_{10}(2 z) \vartheta_{00}(2 w)+\vartheta_{00}(2 z) \vartheta_{10}(2 w), \\
W_{2} & \longmapsto i\left(\vartheta_{10}(2 z) \vartheta_{00}(2 w)-\vartheta_{00}(2 z) \vartheta_{10}(2 w)\right), \\
W_{3} & \longmapsto \vartheta_{00}(2 z) \vartheta_{00}(2 w)-\vartheta_{10}(2 z) \vartheta_{10}(2 w),
\end{aligned}
$$

Hence, the complexified box variety is $\mathcal{B} \cong \overline{\mathbb{H} \times \mathbb{H} / \Delta(4,8)}$.

Proof. The classical theta relations given in Theorem 2.1 show that this is a homomorphism. Obviously, it is surjective. Since $A(\Delta(4,8))$ is an integral domain of Krull dimension three and since $B$ also has dimension three, this homomorphism must be an isomorphism.

The modular picture can be used to recover known properties of the box variety. We mention some of them.

First, we describe the automorphism group of the box variety. The group $\Delta(4,8)$ is a normal subgroup of $\Delta(1,2)$. The index is 768 . Hence, the quotient $\Delta(1,2) / \Delta(4,8)$ is a subgroup of order 768 of the automorphism group. The involution $(z, w) \mapsto(w, z)$ gives an extra automorphism. Both together generate a subgroup of order 1,536 of the automorphism group. By [ST] the order of the automorphism group is 1,536 . Hence, we described the full automorphism group.

Now we describe the singularities. It is known by [ST] that the box variety has 48 singularities, which all are nodes. In the modular picture, they correspond to some zero-dimensional cusps. These are the images of the points $(a, b)$ where $a, b \in \mathbb{Q} \cup\{\infty\}$. There are two types of such points. It may happen that $(a, b)$ is the fixed point of a pair $\left(M_{1}, M_{2}\right)$ of parabolic elements. The typical case is $(\infty, \infty)$ and $A=B=\left(\begin{array}{ll}1 & 4 \\ 0 & 1\end{array}\right)$. The group $\Delta(1,2)$ acts transitively on them. There are pairs that do not have this property. The precise picture is as follows. 
Proposition 2.5. The box variety $\overline{\mathbb{H} \times \mathbb{H} / \Delta(4,8)}$ contains 96 zero-dimensional cusps. They decompose into two orbits of 48 cusps under $\Delta(1,2)$. The orbit containing the image of $(\infty, \infty)$ defines the singular locus.

A slightly different way to see this is to consider the Galois coverings

$$
\overline{\mathbb{H} / \Gamma[8]} \times \overline{\mathbb{H} / \Gamma[8]} \longrightarrow \overline{\mathbb{H} \times \mathbb{H} / \Delta(4,8)} \longrightarrow \overline{\bar{H} / \Gamma[2]} \times \overline{\mathbb{H} / \Gamma[2]}
$$

The covering group of the first cover is $G=\Delta(4,8) / \Gamma[8] \times \Gamma[8] \cong(\mathbb{Z} / 2 \mathbb{Z})^{3}$. The singular points of the box variety are the images of the fixed points of $G$. They agree with the fibers of three zero-dimensional cusps of $\overline{\mathbb{H} / \Gamma[2]} \times \overline{\mathbb{H} / \Gamma[2]}$, which are of the form $(a, a)$. They can be represented by $(\infty, \infty),(0,0)$, and $(1,1)$. In the typical case $(\infty, \infty)$, we can take $p=e^{2 \pi \mathrm{i} z / 8}$ and $q=e^{2 \pi \mathrm{i} w / 8}$ as uniformizing parameters of $\overline{\mathbb{H} / \Gamma[8]} \times \overline{\mathbb{H} / \Gamma[8]}$. The stabilizer in $G$ is generated by the translation $(z, w) \mapsto(z+4, w+4)$, which acts by $(p, q) \mapsto-(p, q)$. Hence, the singularity appears as quotient singularity of the type $(\mathbb{C} \times \mathbb{C}) / \pm$, which actually is a node.

We denote by $\tilde{\mathcal{B}}$ the minimal resolution of the 48 nodes. The exceptional divisor is the union of 48 lines.

Next we describe the holomorphic differential forms on $\tilde{\mathcal{B}}$. The modular curve $\overline{\mathbb{H} / \Gamma[8]}$ has genus 5 . The differentials

$$
\begin{aligned}
& \omega_{1}(z)=\vartheta_{00}(z)^{2} \vartheta_{01}(z) \vartheta_{10}(z) d z, \\
& \omega_{2}(z)=\vartheta_{00}(z) \vartheta_{01}(z)^{2} \vartheta_{10}(z) d z, \\
& \omega_{3}(z)=\vartheta_{00}(z) \vartheta_{01}(z) \vartheta_{10}(z)^{2} d z, \\
& \omega_{4}(z)=\vartheta_{00}(2 z) \vartheta_{00}(z) \vartheta_{01}(z) \vartheta_{10}(z) d z, \\
& \omega_{5}(z)=\vartheta_{10}(2 z) \vartheta_{00}(z) \vartheta_{01}(z) \vartheta_{10}(z) d z
\end{aligned}
$$

are holomorphic on $\overline{\mathbb{H} / \Gamma[8]}$ since the defining modular forms are cusp forms of weight two. Moreover, they are independent since they transform with different multipliers with respect to the group $\Gamma[4]$. A simple computation gives that $\psi_{1}=$ $\omega_{1}(z) \wedge \omega_{1}(w), \psi_{2}=\omega_{2}(z) \wedge \omega_{2}(w), \psi_{3}=\omega_{3}(z) \wedge \omega_{3}(w), \psi_{4}=\omega_{4}(z) \wedge \omega_{4}(w)$, $\psi_{5}=\omega_{4}(z) \wedge \omega_{5}(w), \psi_{6}=\omega_{5}(z) \wedge \omega_{4}(w), \psi_{7}=\omega_{5}(z) \wedge \omega_{5}(w)$ are $\Delta(4,8)-$ invariant holomorphic differential forms on $\mathcal{B}$. We can check that they extend holomorphically to the desingularization $\tilde{\mathcal{B}}$. In this way, we can recover the result of [ST] that the minimal resolution of the box variety has geometric genus 7 . We can also derive from this picture that the box variety is of general type.

In the paper [ST], the structure of the Picard group of $\tilde{\mathcal{B}}$ has been determined. It is a free Abelian group of rank 64. Stoll and Testa proved that certain 140 curves defined already in [vL] generate this group. There are 80 rational and 60 elliptic curves. Of the 80 rational curves, 48 are the exceptional curves described before, whereas the remaining 32 have the following easy modular description.

Proposition 2.6. The equations $w=M z+k$, where $M$ runs through a system of representatives of $\Gamma[4] / \Gamma[8]$ and $k \in\{0,2,4,6\}$, define 32 smooth rational 
curves in the box variety. Their union is the zero set of the modular form

$$
\vartheta_{00}(z)^{4} \vartheta_{01}(w)^{4}-\vartheta_{01}(z)^{4} \vartheta_{00}(w)^{4} \quad\left(=4 \mathrm{i} W_{1} W_{2} W_{3} C\right) .
$$

Proof. We observe that the diagonal $w=z$ is in the zero set of the modular form. The same is true for all conjugated curves. In this way, we see that the modular form in the proposition vanishes at the described 32 curves. Moreover, the zero set of each of $W_{1}, W_{2}, W_{3}, C$ consists of eight rational curves, as we can easily verify by means of the defining equations of the box variety.

Next, we describe the elliptic curves. Part of them is in the Satake boundary. The Satake boundary is the union of the images of $\mathbb{H}^{*} \cup\{a\}$ and $\{a\} \cup \mathbb{H}^{*}$, where $a \in \mathbb{Q} \cup\{\infty\}$. It is easy to work out the structure.

Proposition 2.7. The Satake boundary consists of 12 (smooth) elliptic curves. Each of them contains eight singular and eight smooth zero-dimensional cusps. The whole Satake boundary is the zero set of the modular form

$$
\vartheta_{00}(z) \vartheta_{10}(z) \vartheta_{01}(z) \vartheta_{00}(w) \vartheta_{10}(w) \vartheta_{01}(w) \quad\left(=Z_{1} Z_{2} Z_{3}\right)
$$

We do not give the details of the proof, but we explain a typical boundary curve. We take the image of $\mathbb{H}^{*} \times\{\infty\}$. This is the modular curve with respect to the group $\Gamma_{1}[8] \cap \Gamma[4]$. It contains $\Gamma[8]$ as a subgroup of index 2 . It is not difficult to work out the structure of the ring of modular forms. Details can be found in [K1].

Proposition 2.8. The ring $A\left(\Gamma_{1}[8] \cap \Gamma[4]\right)$ of all modular forms of half integral weight for the group $\Gamma_{1}[8] \cap \Gamma[4]$ with respect to the multiplier system $v_{\vartheta}^{r}$ is generated by

$$
a=\vartheta_{0,0}(z), \quad b=\vartheta_{0,1}(z), \quad c=\vartheta_{0,0}(2 z), \quad d=\vartheta_{1,0}(2 z) .
$$

Defining relations are

$$
a^{2}=c^{2}+d^{2}, \quad b^{2}=c^{2}-d^{2} .
$$

This is an intersection of two quadrics in $\mathbb{P}^{3}$ and hence an elliptic curve. So this describes one of the 12 elliptic curves in the Satake boundary of the box variety of the box variety.

Finally, we describe 48 elliptic curves that are not contained in the boundary.

Proposition 2.9. The equation $w=z+1$ describes an elliptic curve in the box variety, which also can be defined by the equations

$$
\begin{aligned}
W_{1} & =W_{2}, \quad Z_{1}=Z_{2}, \quad \sqrt{2} W_{1}=Z_{3}, \\
W_{3}^{2}+Z_{3}^{2}-C^{2} & =0, \quad 2 Z_{2}^{2}+Z_{3}^{2}-2 C^{2}=0 .
\end{aligned}
$$

Applying the group $\Delta(1,2)$, we get 48 elliptic curves.

The 92 curves, given in Propositions 2.6, 2.7, 2.9, coincide with those in [ST], as can be seen from their description in the box-coordinates. Hence, as has been 
proved in [ST], together with the 48 exceptional curves, they generate the Picard group.

\section{Curves in the Box Variety}

We want to study irreducible curves $C \subset \mathcal{B}$ in the box variety. We have to make the rather strong assumption that the normalization map $\bar{C} \rightarrow C$ is bijective. We denote by $g$ the genus of $\bar{C}$. We use the following fact, which has been explained in [ST] and [Be] and which can be seen from the given explicit description of the holomorphic 2 -forms on $\tilde{\mathcal{B}}$ : the canonical map (defined by the canonical divisor on $\tilde{\mathcal{B}}$ ) is the composition of the natural projection $\tilde{\mathcal{B}} \rightarrow \mathcal{B}$ and the original embedding $\mathcal{B} \rightarrow \mathbb{P}^{6}$. This shows that the degree $d$ of $C$ in $\mathbb{P}^{6}$ equals the intersection number of the strict transform of $C$ in $\tilde{\mathcal{B}}$ and a canonical divisor on $\tilde{\mathcal{B}}$.

Theorem 3.1. Let $C \subset \mathcal{B}$ be a curve such that the normalization map $\bar{C} \rightarrow C$ is bijective. Let $g$ be the genus of $\bar{C}$, and $d$ be the degree of $C$. Then we have the inequality

$$
d \leq 176+16 g
$$

As a consequence, rational and elliptic curves have bounded degree. This supports a conjecture in [ST] that the only rational or elliptic curves contained in $\mathcal{B}$ are the 140 curves described previously.

Proof of Theorem 3.1. Let $k$ be a natural number. We consider a modular form of weight $4 k$ for the group $\Delta(4,8)$. Then we consider the tensor

$$
T=\Delta(z)^{k} \Delta(w)^{k} f(z, w)(d z d w)^{8 k}
$$

in the algebra of symmetric tensors. Since the modular form $\Delta$ has weight 12 , this tensor is invariant under $\Delta(4,8)$. Hence, it defines a meromorphic tensor on $\tilde{\mathcal{B}}$. Using the coordinates $p=e^{2 \pi \mathrm{i} z / 8}, q=e^{2 \pi \mathrm{i} w / 8}$, it is easy to check that this tensor is holomorphic outside the 48 exceptional curves. In the exceptional curves, it may have poles. We can lift the curve $\bar{C}$ to a holomorphic map $\varphi: \bar{C} \rightarrow \tilde{\mathcal{B}}$. Then we consider the pulled back tensor $\varphi^{*} T$. This is a meromorphic tensor of degree $16 k$ on $\bar{C}$.

We can assume that $C$ is not the image of $\mathbb{H}^{*} \times\{a\}$ or $\{a\} \times \mathbb{H}^{*}$ for $a \in \mathbb{H}^{*}$ since for these curves the theorem can be seen directly. Then the tensor $\varphi^{*} T$ vanishes if and only $f$ vanishes along $C$ as a function. Since the weight $k$ can be made large, we can choose $f$ that does not vanish along $C$, and in addition we can get that $f$ does not vanish at any of the 48 nodes in $\mathcal{B}$.

We assume that $C$ contains one of the nodes, for example, the image of the cusp $(\infty, \infty)$. We recall that this point is fixed by the translations. Then we can consider a parameterization of the curve close to this cusp. So we have a pointed disk contained in $C \backslash(\infty, \infty)$. In [Fr1, Satz 1], it is described how a complex curve in a Hilbert modular variety can run into a cusp. For this purpose, such a curve has been described close to the $\operatorname{cusp}(\infty, \ldots, \infty)$ by a holomorphic map 
$\alpha: \mathbb{H} \rightarrow \mathbb{H} \times \cdots \times \mathbb{H}$. The same argument works in our split case and shows that

$$
\alpha(z)=z\left(a_{1}, a_{2}\right)+\left(\Phi_{1}(q), \Phi_{2}(q)\right), \quad q=e^{2 \pi \mathrm{i} \tau},
$$

where $\Phi_{i}$ are holomorphic at $q=0$. The pair $\left(a_{1}, a_{2}\right)$ is contained in the translation lattice, that is,

$$
a_{1} \equiv a_{2} \equiv 0 \bmod 4, \quad a_{1}+a_{2} \equiv 0 \bmod 8 .
$$

The numbers $a_{1}, a_{2}$ are both positive. This implies $a_{1}+a_{2} \geq 8$.

We study the poles and zeros of the tensor $\varphi^{*} T$. Since its divisor is the $16 k$ multiple of a canonical divisor, we have

$$
16(2 g-2) k=\text { \#zeros }- \text { \#poles. }
$$

First, we estimate the number of poles of $\varphi^{*} T$ from above (counted with multiplicity). The poles are intersection points of $\bar{C}$ with the exceptional divisor. Since $\bar{C} \rightarrow C$ is bijective, $\bar{C}$ can meet each of the 48 exceptional curves at most once. Hence, the set of poles contains at most 48 points.

We have to estimate the pole order. It is sufficient to do this for the standard node, that is, the image of the cusp $(\infty, \infty)$. Using the parameterization $\alpha(z)$, we can see that the term $(d z d w)^{8 k}$ contributes with $16 k$ to the pole order and $\Delta(z) \Delta(w)$ contributes with a zero of order $\left(a_{1}+a_{2}\right) k \geq 8 k$. Hence, the pole order of the tensor at the node is at most $8 k$. Since we have 48 nodes, the total pole order is at most $384 k$.

Next, we estimate the number of zeros from below. Each intersection point of the zero divisor of $f$ with the curve produces a zero. (Since we assumed that $f$ does not vanish at the nodes, there is no cancellation between poles and zeros of $T$.) Since the zero divisor of $f$ is a $2 k$-multiple of the canonical divisor, we get that there are at least $2 k d$ zeros. So we get

$$
16(2 g-2) k=\text { \#zeros }- \text { \#poles } \geq 2 k d-384 k .
$$

This finishes the proof of Theorem 3.1.

REMark. As we mentioned, the tensor $T=\Delta(z)^{k} \Delta(w)^{k} f(z, w)(d z d w)^{8 k}$ can have poles along the 48 exceptional divisors. The results of Theorem 3.1 could be improved if one could find $f$ such that $T$ is holomorphic on the whole $\tilde{\mathcal{B}}$. We did not succeed to find such $f$, and we think that they do not exist, although we could not prove this.

\section{A Two-Fold Covering of the Box Variety}

We consider the subgroup $\Gamma^{\prime}[4]$ of index two of $\Gamma[4]$, which is generated by $\Gamma[8], T T^{\prime}$, and $T R$. As can be seen via an explicit calculation in the finite group $\mathrm{SL}(\mathbb{Z} / 8 \mathbb{Z})$, this group is equal to the subgroup of $\Gamma[4]$ given by the condition

$$
a+b+c \equiv 1 \bmod 8 .
$$

LEMma 4.1. The group $\Gamma^{\prime}[4] / \Gamma[8]$ is isomorphic to $\mathbb{Z} / 2 \mathbb{Z} \times \mathbb{Z} / 2 \mathbb{Z}$. It acts freely on $\overline{\mathbb{H} / \Gamma[8]}$. 
Proof. Let $a$ be a point of the extended upper half-plane $\mathbb{H}^{*}=\mathbb{H} \cup \mathbb{Q} \cup\{\infty\}$. Assume that $M \in \Gamma^{\prime}[4]-\Gamma[8]$ is a matrix that fixes $a \bmod \Gamma[8]$. Then there exists an element $A \in \Gamma[8]$ such that $M(a)=A(a)$. The matrix $N=A^{-1} M$ fixes $a$. This matrix is also contained in $\Gamma^{\prime}[4]$ and not in $\Gamma[8]$. Modulo 8 it is one of the following three matrices:

$$
\left(\begin{array}{ll}
1 & 4 \\
4 & 1
\end{array}\right), \quad\left(\begin{array}{ll}
5 & 4 \\
0 & 5
\end{array}\right), \quad\left(\begin{array}{ll}
5 & 0 \\
4 & 5
\end{array}\right) .
$$

Since it has a fixed point, the absolute value of its trace is bounded by 2 . We treat the three cases separately. In the first case, we have

$$
N=\left(\begin{array}{ll}
1+8 \alpha & 4+8 \beta \\
4+8 \gamma & 1+8 \delta
\end{array}\right) .
$$

The condition for the trace implies $\delta=-\alpha$. The determinant is 1 . But the condition $\delta=-\alpha$ implies that the determinant is $1-16 \bmod 32$. This is a contradiction.

In the second case, we have

$$
N=\left(\begin{array}{cc}
5+8 \alpha & 4+8 \beta \\
8 \gamma & 5+8 \delta
\end{array}\right) .
$$

The condition for the trace now gives $\delta=-\alpha-1$. Now the determinant would be congruent to $25-40=17 \bmod 32$, which is not possible. The same argument works in the third case.

We consider the (nonsingular) manifold

$$
X:=(\overline{\mathbb{H} / \Gamma[8]} \times \overline{\mathbb{H} / \Gamma[8]}) / \Gamma^{\prime}[4],
$$

where $\Gamma^{\prime}[4]$ acts diagonally. The inclusion $\Gamma^{\prime}[4] \hookrightarrow \Gamma[4]$ gives a two-fold covering $X \rightarrow \mathcal{B}$ of the box variety. Locally around the 48 singularities of $\mathcal{B}$, this looks like the covering $\mathbb{C}^{2} \rightarrow \mathbb{C}^{2} / \pm$. We can desingularize the node at 0 by first blowing up the origin in the covering $\mathbb{C}^{2}$. The involution $(z, w) \mapsto(-z,-w)$ lifts to this resolution, and the quotient is smooth. The same can be done globally. We blow up $X$ at the 48 inverse images of the nodes of $\mathcal{B}$. This gives a manifold $\tilde{X}$. The Galois involution of $X$ over $\mathcal{B}$ lifts to $\tilde{X}$, and the quotient $\tilde{\mathcal{B}}$ is just the blow up of $\mathcal{B}$ at the nodes. So we have a commutative diagram

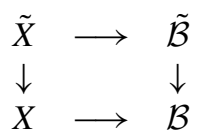

The map $\tilde{X} \rightarrow \tilde{\mathcal{B}}$ is ramified along the exceptional divisors (48 lines). The existence of this covering and its uniqueness have been treated in [Be]. We call

$$
X=(\overline{\mathbb{H} / \Gamma[8]} \times \overline{\mathbb{H} / \Gamma[8]}) / \Gamma^{\prime}[4]
$$

the Beauville manifold. The existence of $X$ is equivalent to the fact that the exceptional divisor is divisible by two in the Picard group $\operatorname{Pic}(\tilde{\mathcal{B}})$. The uniqueness follows from the fact that this Picard group is torsion free [ST]. We refer to [Be] for more interesting properties of the surface $X$. Some of them can be easily derived from the modular picture. 
Since the universal covering of $X$ is the product of two half-planes, it cannot contain a rational or elliptic curve. So we obtain the following result.

REMARK 4.2. Every rational or elliptic curve in the box variety contains at least one node.

\section{Relation to a Kummer Variety}

In this section, we consider the $\mathbb{Q}$-structure of the box variety. It is the projective variety associated with the algebra

$$
B=\mathbb{Q}\left[W_{1}, W_{2}, W_{3}, Z_{1}, Z_{2}, Z_{3}, C\right]
$$

(with the defining relations of the box variety). We consider the involution $\sigma\left(Z_{3}\right)=-Z_{3}$ of the ring $B$. It induces an involution of the box variety. The invariant ring is

$$
B^{\sigma}=\mathbb{Q}\left[Z_{1}, Z_{2}, C, W_{1}, W_{2}, W_{3}\right]
$$

with defining relations

$$
\begin{aligned}
W_{1}^{2}+W_{3}^{2} & =Z_{2}^{2}, \\
W_{2}^{2}+W_{3}^{2} & =Z_{1}^{2}, \\
W_{1}^{2}+W_{2}^{2}+W_{3}^{2} & =C^{2} .
\end{aligned}
$$

The associated projective variety is the quotient of the box variety by $\sigma$. This is a also a modular variety since in the picture of Theorem 2.4, the transformation $\sigma$ is induced by the modular substitution

$$
(z, w) \longmapsto(T z, w) .
$$

This gives the following result.

Lemma 5.1. The variety $\mathcal{B} / \sigma$ is defined over $\mathbb{Q}$. Over $\mathbb{C}$, it agrees with the modular variety defined by the subgroup of $\operatorname{SL}(2, \mathbb{Z}) \times \operatorname{SL}(2, \mathbb{Z})$ that is generated by $\Gamma[8] \times \Gamma[8]$ and the elements

$$
(T, E), \quad(E, T), \quad\left(T^{\prime}, T^{\prime}\right), \quad(R, R) .
$$

We already considered in Proposition 2.8 the group $\Gamma_{1}[8] \cap \Gamma[4]$ and explained the structure of the ring of modular forms. We defined four generators $a, b, c, d$ with defining relations

$$
a^{2}=c^{2}+d^{2}, \quad b^{2}=c^{2}-d^{2} .
$$

Since these relations are defined over $\mathbb{Q}$, we can consider this algebra over $\mathbb{Q}$

$$
A\left(\Gamma_{1}[8] \cap \Gamma[4]\right)=A_{0} \otimes_{\mathbb{Q}} \mathbb{C}, \quad A_{0}:=\mathbb{Q}[a, b, c, d],
$$

and obtain an elliptic curve $E$ over $\mathbb{Q}$. We can compute its normal form over $\mathbb{Q}$ :

$$
y^{2}=x^{3}-x .
$$

Its projective form is $y^{2} z=x^{3}-x z^{2}$. An explicit isomorphism is given by

$$
x=a-b, \quad y=2 d, \quad z=2 c-a-b .
$$


We consider the automorphisms

$$
\tau(a, b, c, d)=(a,-b, c, d), \quad \rho(a, b, c, d)=(a, b,-c,-d)
$$

of the algebra. They correspond to the modular transformations $T^{\prime}$ and $R$, whereas $T$ acts as the identity. The two transformations generate a group $H \cong \mathbb{Z} / 2 \mathbb{Z} \times$ $\mathbb{Z} / 2 \mathbb{Z}$.

Lemma 5.2. The transformation $\rho$ is an involution without fixed point of the elliptic curve E. Hence, it is a translation by a two-torsion point. The transformation $\tau$ is an involution with the fixed point $[\sqrt{2}, 0,1,1]$. Hence, the following is true. If we consider $E$ as an elliptic curve over $\mathbb{Q}(\sqrt{2})$ with origin $[\sqrt{2}, 0,1,1]$, then $\tau$ corresponds to the map " $x \mapsto-x$ ”.

We want to consider the product of two copies of this curve. This is the projective variety associated with the graded algebra

$$
A_{2}=\mathbb{Q}[a \otimes a, a \otimes b, \ldots, d \otimes d] .
$$

In the modular picture, we have to identify

$$
a \otimes a=\vartheta_{00}(z) \vartheta_{00}(w), \ldots, d \otimes d=\vartheta_{10}(2 z) \vartheta_{10}(2 w) .
$$

The group $H$ acts diagonally on $A_{2}$. The ring of invariants is just $B^{\sigma}$. Hence, we get the following result.

Proposition 5.3. There is a biholomorphic map

$$
(E \times E) / H \stackrel{\sim}{\longrightarrow} \mathcal{B} / \sigma,
$$

defined over the field of Gauss numbers.

The variety $(E \times E) / H$ can be understood as follows. We first take the quotient by the translation $\varrho$. This gives an Abelian variety over $\mathbb{Q}$,

$$
X=(E \times E) / \varrho .
$$

Then we take the quotient by $\tau$. If we extend the base field $\mathbb{Q}$ by $\sqrt{2}$ and take $[\sqrt{2}, 0,1,1]$ diagonally embedded as the origin, then $\tau$ corresponds to the negation, and

$$
(E \times E) / H=X / \pm
$$

appears as a Kummer variety. Hence, over the field $\mathbb{Q}(i, \sqrt{2})$ of eighth roots of unity, the variety $\mathcal{B} / \sigma$ can be identified with a Kummer variety.

\section{A Moduli Problem}

In this section, we give a modular interpretation of the variety $(\mathbb{H} \times \mathbb{H}) / \Delta\left(N, N^{\prime}\right)$. First, we recall from [KM] some basic facts. We denote by $(\mathrm{Sch} / S)$ the category of schemes over a base scheme $S$. For $S=\operatorname{Spec} A$, we write $(\operatorname{Sch} / A)$. We fix an algebraic number field $K$. We also fix an embedding $K \hookrightarrow \mathbb{C}$. The category 
$($ Ell $)=($ Ell $) / K$ has as objects elliptic curves $\pi: E \rightarrow S$ over a variable basescheme $S \in(\mathrm{Sch} / K)$, and its morphisms are Cartesian squares of elliptic curves. A contravariant functor

$$
\mathcal{P}:(\text { Ell }) \longrightarrow \text { (Sets) }
$$

is called a moduli problem for elliptic curves. The moduli problem is called relatively representable over (Ell) if for every elliptic curve $E / S$, the functor on $\mathrm{Sch} / S$ defined by

$$
T \longmapsto \mathcal{P}\left(E_{T} / T\right)
$$

is representable by an $S$-scheme denoted by $\mathcal{P}_{E / S}$. The moduli problem is called representable if there exists an elliptic curve over a scheme

$$
\mathbf{E} \longrightarrow \mathcal{M}(\mathcal{P})
$$

together with a functorial isomorphism

$$
\mathcal{P}(E / S) \stackrel{\sim}{\longrightarrow} \operatorname{Hom}_{(\mathrm{Ell})}(E / S, \mathbf{E} / \mathcal{M}(\mathcal{P})) .
$$

If the moduli problem $\mathcal{P}$ is representable, then the scheme $\mathcal{M}(\mathcal{P})$ represents the functor over (Sch)

$$
S \longmapsto\{\text { isomorphism classes of }(E / S, \alpha)\} .
$$

Here $E$ is an elliptic curve over $S$, and $\alpha \in \mathcal{P}(E / S)$. Any representable moduli problem $\mathcal{P}$ is relatively representable.

If $\mathcal{P}^{\prime}$ is representable and $\mathcal{P}^{\prime \prime}$ is relatively representable, then the simultaneous moduli problem $\left(\mathcal{P}^{\prime}, \mathcal{P}^{\prime \prime}\right)$

$$
E / S \longmapsto \mathcal{P}^{\prime}(E / S) \times \mathcal{P}^{\prime \prime}(E / S)
$$

is representable. In fact, if $\mathbf{E} / \mathcal{M}\left(\mathcal{P}^{\prime}\right)$ represents $\mathcal{P}^{\prime}$, then

$$
\mathcal{M}\left(\mathcal{P}^{\prime}, \mathcal{P}^{\prime \prime}\right)=\mathcal{P}_{\mathbf{E} / \mathcal{M}\left(\mathcal{P}^{\prime}\right)}^{\prime \prime}
$$

Let $E / S$ be an elliptic curve, and $N$ be a natural number. We denote by $E[N]$ the kernel of multiplication by $N$ from $E$ to $E$. This is a group scheme over $S$. If $T$ is a scheme over $S$, then the $T$-valued points are

$$
E[N](T)=\operatorname{kernel}(E(T) \stackrel{\cdot N}{\longrightarrow} E(T)) .
$$

It may be that $E[N](S)$ is isomorphic $(\mathbb{Z} / N \mathbb{Z} \times \mathbb{Z} / N \mathbb{Z})(S)$. A level $N$ structure on $E$ then means the choice of an isomorphism

$$
(\mathbb{Z} / N \mathbb{Z} \times \mathbb{Z} / N \mathbb{Z})(S) \stackrel{\sim}{\longrightarrow} E[N](S) .
$$

It extends to an isomorphism of $E(N)$ to the constant group scheme associated with $\mathbb{Z} / N \mathbb{Z} \times \mathbb{Z} / N \mathbb{Z}$.

The kernel of the $N$ th power is the group scheme $\mu_{N}$. Hence,

$$
\mu_{N}(S)=\left\{a \in \mathcal{O}(S) ; a^{N}=1\right\} .
$$

For an elliptic curve $E$ over $S \in(\mathrm{Sch} / K)$, there exists the Weil pairing. It associates with each $S$-scheme $T$ an alternating map

$$
E[N](T) \times E[N](T) \longrightarrow \mu_{N}(T) .
$$


When $K$ contains the cyclotomic field of $N$ th roots of unity, $\mu_{N}$ is the constant group scheme associated with the abstract group

$$
\mu_{N}(\mathbb{C})=\left\{\zeta \in \mathbb{C} ; \zeta^{N}=1\right\} \cong \mathbb{Z} / N \mathbb{Z} .
$$

We also can consider the symplectic pairing

$$
\begin{aligned}
& e_{N}: \mathbb{Z} / N \mathbb{Z} \times \mathbb{Z} / N \mathbb{Z} \longrightarrow \mu_{N}(\mathbb{C}), \\
& \quad e\left(\left(a_{1}, a_{2}\right),\left(b_{1}, b_{2}\right)\right)=e^{2 \pi \mathrm{i}\left(a_{1} b_{2}-a_{2} b_{1}\right) / N} .
\end{aligned}
$$

From now on we assume that $K$ contains the $N$ th roots of unity. Since we consider a fixed embedding of $K$ into $\mathbb{C}$, we can identify $\mu_{N}(\mathbb{C})$ and $\mu_{N}(K)$. So it makes sense to consider level $N$ structures that preserve the symplectic pairings.

We denote by $\mathcal{P}_{N}^{\prime}$ the moduli problem that classifies the level $N$ structures preserving the symplectic pairings. It is known (see [KM]) that this moduli problem is representable if $N \geq 3$.

We formulate a second moduli problem $\mathcal{P}_{N}^{\prime \prime}$ on (Ell),

$$
\mathcal{P}_{N}^{\prime \prime}(E / S)=\text { Isomorphy classes of pairs }(F / S, \beta),
$$

where $\beta: E[N] \stackrel{\sim}{\longrightarrow} F[N]$ is an isomorphism of $S$-groups that preserves the Weil pairings. This is a moduli problem studied in [FK]. There has been proved that it is relatively representable, quasi-affine, smooth, and geometrically connected of relative dimension 1 if $N \geq 3$. We now consider two natural numbers $N \mid N^{\prime}$ such that $N \geq 3$. The simultaneous problem

$$
\mathcal{P}\left(N, N^{\prime}\right)=\left(\mathcal{P}_{N}^{\prime}, \mathcal{P}_{N^{\prime}}^{\prime \prime}\right)
$$

is representable. Hence, we can summarize with the following theorem.

THeOREM 6.1. Let us assume that $N \geq 3$. The moduli problem $\mathcal{P}\left(N, N^{\prime}\right)$ is representable as a moduli problem on (Ell).

We denote the moduli space of $\mathcal{P}\left(N, N^{\prime}\right)$ by $\mathcal{M}\left(N, N^{\prime}\right)$. This is an affine algebraic scheme over the cyclotomic field $\mathbb{Q}\left(\zeta_{N}\right)$.

Lemma 6.2. The scheme $\mathcal{M}\left(N, N^{\prime}\right) \otimes_{K} \mathbb{C}$ can be identified with $(\mathbb{H} \times \mathbb{H}) /$ $\Delta\left(N, N^{\prime}\right)$.

This is a generalization of the well-known fact that $\mathbb{H} / \Gamma[q]$ parameterizes elliptic curves over $\mathbb{C}$ with a level $q$ structure. In the case $N=1$, the variety $\mathcal{M}\left(N, N^{\prime}\right)$ is a particular case of the diagonal quotient surfaces $\mathcal{Z}_{N^{\prime}, \varepsilon}[\mathrm{KS}]$, which, over $\mathbb{C}$, have been introduced by Hermann [He] in 1991. The proof in [KS] works for arbitrary $N$. We can omit it here.

We are interested in the group $\Delta(4,8)$. Theorem 2.4 says that the surface $(\mathbb{H} \times \mathbb{H}) / \Delta(4,8)$ is embedded as an open part of the box variety. We call this the finite part of the box variety. We have now two $\mathbb{Q}(\mathrm{i})$-structures, one coming from the defining equations of the box variety and the other coming from the moduli problem $\mathcal{P}(4,8)$. 
THEOREM 6.3. The variety $\mathcal{M}(4,8)$, as a variety over $\mathbb{Q}\left(\zeta_{8}\right)$, is isomorphic to the finite part of the box variety (considered as a variety over $\mathbb{Q}\left(\zeta_{8}\right)$ ).

Proof. Let $K=\mathbb{Q}\left(\zeta_{8}\right)$. The Galois group $\operatorname{Gal}(\mathbb{C} / K)$ acts on $\mathcal{M}(4,8) \otimes_{K} \mathbb{C}$ and hence on $(\mathbb{H} \times \mathbb{H}) / \Delta(4,8)$. We have to show that the seven modular functions (generators of the field of modular functions) corresponding to $Z_{2} / Z_{1}, \ldots, W_{3} / Z_{1}, C / Z_{1}$ (see Theorem 2.4) commute with the action of the Galois group. For example, $Z_{2} / Z_{1}$ corresponds to

$$
\frac{\vartheta_{00}(z)}{\vartheta_{01}(z)} \frac{\vartheta_{00}(w)}{\vartheta_{01}(w)} \text {. }
$$

So it is sufficient to prove that $\vartheta_{00}(z) / \vartheta_{01}(z)$, considered as a rational function on the modular curve $X(8)$ (see [DR]) commutes with the action of this Galois group. Since this modular function has rational Fourier coefficients, it is defined over $K$ as follows from the $q$-expansion principle in [DR].

From Theorem 6.3 we obtain the following result.

THEOREM 6.4. The $\mathbb{Q}\left(\zeta_{8}\right)$-valued points of the finite part of the box variety are in one-to-one correspondence with the isomorphy classes of pairs of elliptic curves $E, F$ over $\mathbb{Q}\left(\zeta_{8}\right)$, equipped with a level 4 structure and a compatible isomorphism of group schemes $E[8] \rightarrow F[8]$ that preserves the Weil pairing.

\section{References}

[Be] A. Beauville, A tale of two surfaces, 2013, arXiv:1303.1910v1.

[Br] S. Brieger, Modulformen zur Stufe 8, Diplomarbeit, Universität Heidelberg, 2010.

[DR] P. Deligne and M. Rapoport, Les schémas de modules de courbes elliptiques, Proc. Antwerpen conference, vol. 2, Lecture Notes in Math., 349, pp. 143-316, SpringerVerlag, Berlin, 1973.

[Fr1] E. Freitag, Eine Bemerkung zur Theorie der Hilbertschen Modulmannigfaltigkeiten hoher Stufe, Math. Z. 171 (1980), 27-38.

[Fr2] , Siegelsche Modulfunktionen, Grundlehren Math. Wiss., 254, SpringerVerlag, Berlin, 1983.

[FK] G. Frey and E. Kani, Curves of genus 2 with elliptic differential and associated Hurwitz space, Contemp. Math. 487 (2009), 33-81.

[He] C. F. Hermann, Modulfä̈chen quadratischer Diskriminante, Manuscripta Math. 72 (1991), 95-110.

[KS] E. Kani and W. Schanz, Modular diagonal quotient surfaces, Math. Z. 227 (1998), 337-366.

[KM] N. M. Katz and B. Mazur, Arithmetic moduli of elliptic curves, Ann. of Math. Stud., Princeton University Press, Princeton, NJ, 1985.

[Kl] M. Klein, Ein Ring von Modulformen zur Stufe 8, Diplomarbeit, Universität Heidelberg, 2011.

[ST] M. Stoll and D. Testa, The surface parametrizing cuboids, preprint, arXiv:1009.0388.

[vGN] B. Van Geemen and N. O. Nygaard, L-functions of some Siegel modular 3-folds, J. Number Theory 53 (1995), 45-87.

[vL] R. Van Luijk, On perfect cuboids, Undergraduate thesis, University Utrecht, 2000. 
E. Freitag

Mathematisches Institut

Universitat Heidelberg

Im Neuenheimer Feld 288

D 69120 Heidelberg

Germany

freitag@mathi.uni-heidelberg.de
R. Salvati Manni

Dipartimento di Matematica

Università La Sapienza

Piazzale Aldo Moro 2

I 00185 Roma

Italy

salvati@mat.uniroma1.it 